\title{
OS PRINCIPAIS ATIVOS USADOS NA PREVENÇÃO E TRATAMENTO DO MELASMA
}

\author{
THE MAIN ASSETS USED IN THE PREVENTION AND TREATMENT OF MELASMA
}

\author{
Cristina Guimarães Santos ${ }^{1}$ \\ Daniele Santa Rosa Bitencourt ${ }^{2}$ \\ Lívia Gomes de Brito ${ }^{3}$ \\ José Fernando de Araújo Neto 4
}

RESUMO: O melasma é uma alteração na pigmentação da pele, que provoca manchas, principalmente na face, gerando impacto negativo na vida dos pacientes. $O$ uso de dermocosméticos e sua associação com fotoprotetores promovem a melhora no âmbito estético, permitindo que o paciente usufrua de uma boa qualidade de vida. Objetivo: $\mathrm{O}$ presente estudo tem como objetivo buscar as principais formas de prevenção e tratamento do melasma, a partir do uso de dermocosméticos e fotoprotetores. Métodos: Foi realizada uma revisão bibliográfica integrativa por meio da busca de artigos nas bases de dados: Google Scholar, LILACS e SciELO, resultando em 25 artigos científicos. Resultados: Foram descritos em tabelas pontos importantes dos artigos: autores/ano, objetivo (s) e resultados/conclusão. A discussão foi realizada de forma descritiva, tendo os estudos selecionados em consonância com o objetivo proposto. Considerações finais: Destaca-se o uso dos ácidos glicólico, kójico e mandélico que apresentaram resultados positivos no clareamento da pele, conferindo melhora na qualidade de vida dos pacientes. Contudo, é fundamental empregar o uso de fotoprotetores para prevenção, juntamente com o tratamento facial adequado.

Palavras-chave: Hipercromias. Dermocosméticos. Fotoprotetores.

ABSTRACT: Melasma is a change in skin pigmentation, which causes spots, especially on the face, generating a negative impact on patients' lives. The use of dermocosmetics and their association with photoprotectors promote improvement in the aesthetic sphere, allowing the patient to enjoy a good quality of life. Objective: This study aims to seek the main forms of prevention and treatment of melasma, from the use of dermocosmetics and photoprotectors. Methods: An integrative literature review was carried out by searching for articles in the following databases: Google Scholar, LILACS and SciELO, resulting in 25

\footnotetext{
${ }^{1}$ Acadêmica do curso de Farmácia da Universidade Salvador - UNIFACS - Salvador-BA.Email:cristinabio3@hotmail.com.

${ }^{2}$ Acadêmica do curso de Farmácia da Universidade Salvador - UNIFACS - Salvador-BA.E-

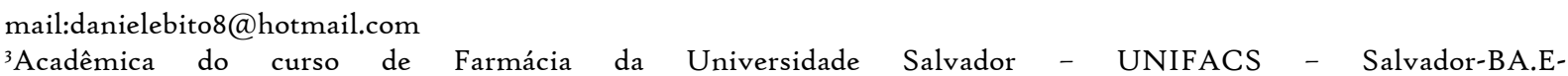
mail:livinhagomesb@hotmail.com

${ }_{4}^{4}$ Graduado em Farmácia pela Universidade Federal da Bahia - UFBA; Docente do curso de Farmácia da Universidade Salvador - UNIFACS - Salvador - Bahia. E-mail: jose.araujo@unifacs.br.
} 
scientific articles. Results: Important points of the articles were described in tables: authors/year, objective(s) and results/conclusion. The discussion was conducted descriptively, and the studies were selected in line with the proposed objective. Final considerations: The use of glycolic, kojic and mandelic acids that showed positive results in skin whitening, improving the quality of life of patients, is highlighted. However, it is essential to use photoprotectors for prevention, along with appropriate facial treatment.

Keywords: Hyperchromia. Dermocosmetics. Photoprotectors.

\section{INTRODUÇÃO}

A condição e a aparência da pele são fundamentais para demonstrar uma aparência saudável, sobretudo, os fatores como o estado da córnea e a pigmentação presente (MACEDO, 2019). A hiperpigmentação da pele pode ter uma característica natural, padrões de herança, problemas de pele e doenças, contudo, estudos mostram que as complicações estão associadas a mudanças no pigmento melânico (CESTARI et al., 2014).

A melanina é um polímero pigmentado que determina ao tecido da pele uma cor acastanhada, especificando que é função da eumelanina, um polímero que varia do marrom ao preto, e das feomelaninas, compostos amarelo-avermelhados, a proteção contra os raios ultravioletas, sendo esta última capaz de gerar radicais livres que ocasionam danos ao DNA e contribuem para os efeitos fototóxicos (CESÁRIO, 2015).

O melasma é uma alteração da pigmentação melânica da pele, adquirida, comum, caracterizada por máculas em geral simétricas, hiperpigmentadas, com margens irregulares e de limites definidos, que ocorre em áreas fotoexpostas e sua principal localização é na face, seguido pelo colo e antebraços (SCHAEFER, 2018).

Entre os fatores de risco para o surgimento do melasma, estão a exposição crônica aos raios ultravioletas - RUV, gestação, terapias hormonais de reposição, contraceptivos orais e alguns esteroides e câncer ginecológico. Portanto o surgimento de pigmentos na

pele além de afetar a imagem de uma pessoa, podem provocar processos relacionados à baixa autoestima (BRIANEZI, 20I6).

O objetivo do tratamento do melasma é o controle da doença, contudo, o diagnóstico clínico é feito com a ajuda do exame da lâmpada de Wood (OLIVEIRA et al., 202I). Ainda assim, a patologia é classificada de acordo com a profundidade da deposição de pigmentos, como: epidérmico (cor torna-se mais acentuada), dérmico (a pigmentação não é 
intensificada com a luz de Wood), misto (a luz de Wood intensifica a pigmentação em algumas áreas, enquanto outras permanecem inalteradas) e inaparente (as manchas são visíveis à luz do dia, e tornam-se inaparentes à luz de Wood) (OLIVEIRA et al., 202I).

Tratamentos para o melasma envolvem o uso de agentes despigmentantes, lasers, peelings químicos, microdermabrasão e até o auxílio de luz intensa pulsada (RODRIGUES, 2014). Certamente, os ácidos aparecem como boas opções de tratamento, pois oferecem renovação celular ou efeito clareador nas manchas. Contudo, a associação entre protetores solares de amplo espectro e cremes despigmentantes caracterizam-se como os dermocosméticos que agem além da epiderme. No entanto, os produtos apresentam as indicações específicas e são desenvolvidos a partir de estudos clínicos e científicos (BORGES, 2018).

Diante do exposto, foram avaliados os artigos disponíveis sobre as principais formas de tratamento do melasma a partir do uso de dermocosméticos e sua associação ao uso dos fotoprotetores. No presente estudo, foi utilizado o método da revisão integrativa de literatura, que visa coletar e sintetizar o conhecimento científico já produzido sobre o tema investigado, permitindo a pesquisa, avaliação e síntese das evidências disponíveis no tratamento do melasma e qualidade de vida.

\section{METODOLOGIA}

Para a elaboração deste estudo, foi realizada uma revisão integrativa de literatura, uma vez que, trata-se de uma análise de estudos importantes, que aprimora a prática e promove o aprendizado em determinados assuntos (BENTO, 2012).

A pesquisa foi realizada por meio de consulta às bases de dados: Google Scholar, Literatura Latino-Americana e do Caribe em Ciências da Saúde (LILACS) e Scientific Electronic Library Online (SciELO), com limitadores temporais no período de publicação de 2011 até 2021.

Para a busca dos artigos, foram utilizados os seguintes descritores e suas combinações na língua portuguesa: hipercromia, profilaxia, agentes despigmentantes, distúrbios pigmentares e ácidos. E na língua inglesa: "hyperchromia, prophylaxis, depigmenting agents, pigmentary disorders, acids, " utilizando como conectivo o termo "and". 
Inicialmente, para que os artigos atendessem a finalidade do estudo, algumas questões foram delimitadas, com vista a obter artigos que abordassem as principais medidas de prevenção e tratamento do melasma, podendo ser pesquisas publicadas em português e/ou inglês.

Os critérios de inclusão definidos para a seleção dos artigos foram pré-selecionados: pelas palavras-chave (filtro $\mathrm{I}$ ), em seguida foram selecionados pelos títulos e objetivo (s) (filtro 2) e para a seleção criteriosa dos artigos foi analisada a metodologia (filtro 3) por fim, os artigos foram lidos na íntegra (filtro 4) e selecionados os que correspondiam aos objetivos deste estudo. Os critérios de exclusão utilizados foram: estudos que não estivessem de acordo com tema em discussão, artigos em idiomas que não fossem os que foram listados anteriormente, documentos relacionados a congressos, conferências e relatórios técnicos, como também, trabalhos publicados que estivessem fora do período mencionado acima (figura I).

Fluxograma r. Representação esquemática da seleção dos artigos.

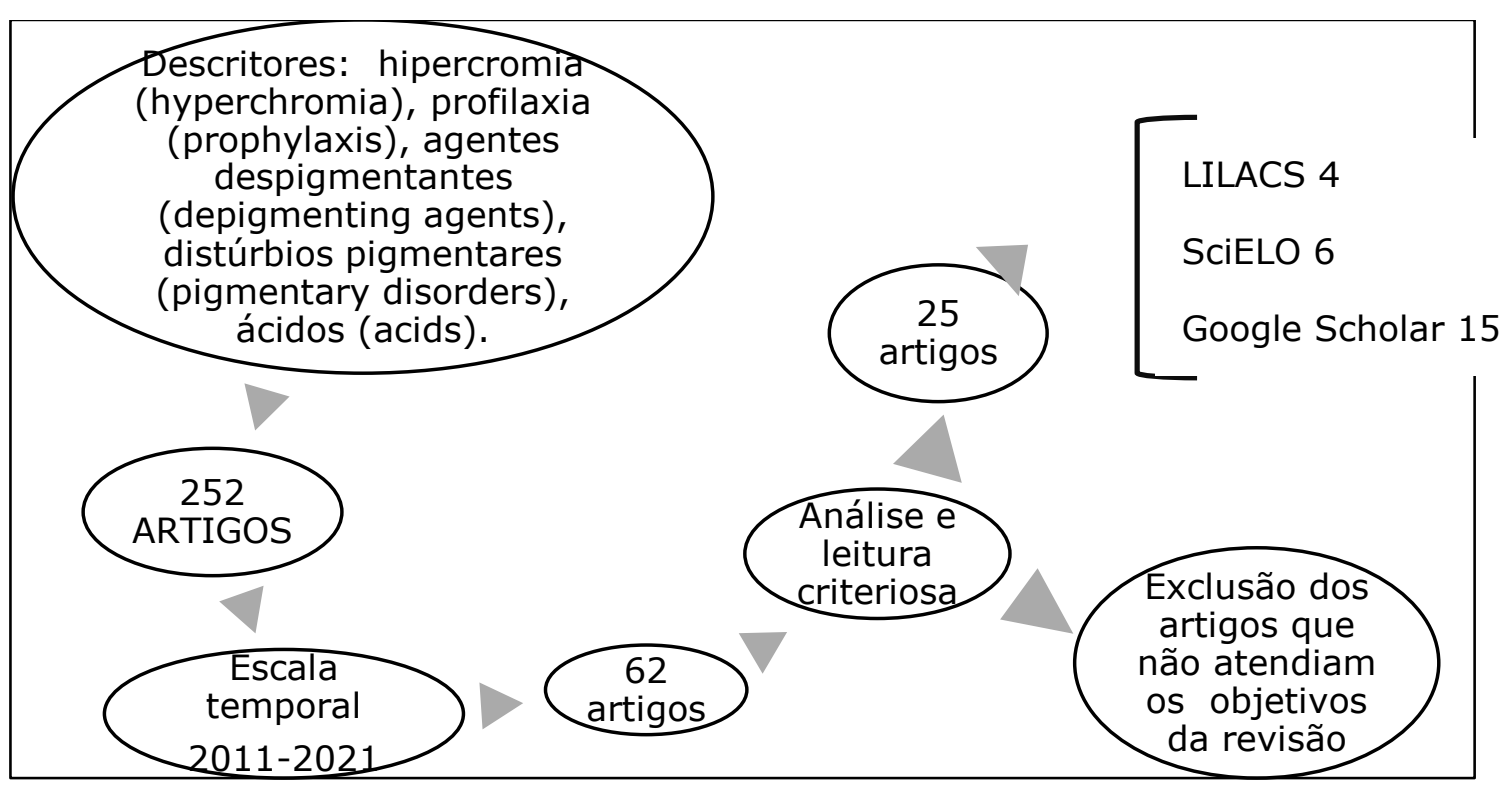

Fonte: elaborada pelos autores (2021)

\section{RESULTADOS}

A pesquisa possibilitou a identificação de 252 artigos separados em, I2 artigos científicos na base de dados do SciELO, representando 4,8\%, no LILACS foram 65 (25.8\%) 
e 175 no Google Scholar (69.4\%). Em síntese, para leitura foram selecionados 62 artigos, porém quando aplicados os critérios de exclusão, uma análise e leitura minuciosa, somente foram utilizados 25 artigos para o desenvolvimento deste trabalho.

Diante da busca nas bases de dados, foram excluídos trabalhos que estivessem fora da escala temporal (20II até 202I), que totalizaram 25 artigos dentro dos critérios de inclusão. Nesta revisão integrativa, após a leitura e análise dos artigos escolhidos, foram considerados o ano de publicação, bem como o objetivo (s) e resultados/conclusão encontrados na tabela IA, ${ }_{\mathrm{IB}}$ e $\mathrm{IC}$.

Das publicações selecionadas, aproximadamente $17,0 \%$ estavam na língua inglesa, no entanto, a maioria das pesquisas encontradas eram estudo de caso e/ou levantamento bibliográfico. Os componentes mais discutidos nos estudos foram: o uso de algum tipo de ácido para o tratamento do melasma $(59,0 \%)$, seguido dos componentes sobre fotoprotetores e caráter socioemocionais abordados $(4 \mathrm{I}, 0 \%)$.

Tabela IA: Síntese dos estudos selecionados para revisão.

\begin{tabular}{|c|c|c|}
\hline Autor/Ano & Objetivo(s) & Resultados/Conclusão \\
\hline $\begin{array}{l}\text { ARAUJO, I. L. } \\
\text { de, MEJIA, D. P. } \\
\text { M.. / } 2014\end{array}$ & $\begin{array}{l}\text { Realizar um levantamento } \\
\text { bibliográfico para a } \\
\text { compreensão do efeito do } \\
\text { peeling químico nas } \\
\text { hipercromias. }\end{array}$ & $\begin{array}{l}\text { Despigmentantes provocam descamação, } \\
\text { renovação celular e melhoram a textura } \\
\text { da pele. A associação entre ácido kójico e } \\
\text { ácido glicólico apresenta efeitos positivos } \\
\text { utilizados no tratamento de hipercromias. }\end{array}$ \\
\hline $\begin{array}{l}\text { BORGES, M. } \\
\text { C../ 202I }\end{array}$ & $\begin{array}{l}\text { Realizar um levantamento } \\
\text { bibliográfico sobre o } \\
\text { tratamento e as implicações } \\
\text { estéticas provocadas pelo } \\
\text { melasma. }\end{array}$ & $\begin{array}{l}\text { O tratamento correto, como o uso } \\
\text { sistemático de várias terapias tópicas, } \\
\text { clareia as manchas na pele, reduzindo a } \\
\text { área afetada, melhorando a qualidade de } \\
\text { vida do paciente. }\end{array}$ \\
\hline $\begin{array}{l}\text { CENTURIÃO, } \\
\text { P. de O, et al./ } \\
\text { 202I }\end{array}$ & $\begin{array}{l}\text { Avaliar a qualidade de } \\
\text { produtos cosméticos de venda } \\
\text { livre contendo ácido glicólico } \\
\text { disponíveis no comércio } \\
\text { nacional. }\end{array}$ & $\begin{array}{l}\text { Fica evidente a importância do controle } \\
\text { de qualidade em produtos contendo ácido } \\
\text { glicólico para conferir segurança e eficácia } \\
\text { para os usuários. }\end{array}$ \\
\hline
\end{tabular}

Fonte: elaborado pelos autores (202I) 
Tabela IB: Síntese dos estudos selecionados para revisão.

\begin{tabular}{|c|c|c|}
\hline Autor/Ano & Objetivo(s) & Resultados/Conclusão \\
\hline $\begin{array}{l}\text { CUNHA, I. G.; } \\
\text { SILVA, C. P. da; } \\
\text { OLIVEIRA, G. B. B. } \\
\text { / } 2020\end{array}$ & $\begin{array}{l}\text { Realizar um levantamento } \\
\text { das principais formas de se } \\
\text { tratar o melasma. }\end{array}$ & $\begin{array}{l}\text { Os ácidos encontrados mais } \\
\text { utilizados são, ácido kójico, } \\
\text { hidroquinona, ácido mandélico, } \\
\text { ácido tranexâmico, entre outros; } \\
\text { não existindo tratamento mais } \\
\text { eficaz que outro. }\end{array}$ \\
\hline $\begin{array}{l}\text { COLFERAI,M.M.T, } \\
\text { MIQUELIN, G.M, } \\
\text { STEINER, D./ } 2018\end{array}$ & $\begin{array}{l}\text { Avaliar a eficácia do ácido } \\
\text { tranexâmico oral no } \\
\text { tratamento do melasma. }\end{array}$ & $\begin{array}{l}\text { O ácido tranexâmico foi eficaz em } \\
50 \% \text { dos pacientes de acordo com os } \\
\text { métodos de avaliação quando } \\
\text { comparado ao placebo. }\end{array}$ \\
\hline DEO et al., /2013 & $\begin{array}{l}\text { Comparar a eficácia do ácido } \\
\text { kójico, com as combinações } \\
\text { hidroquinona ou valerato de } \\
\text { betametasona e dos três } \\
\text { agentes na terapia do } \\
\text { melasma. }\end{array}$ & 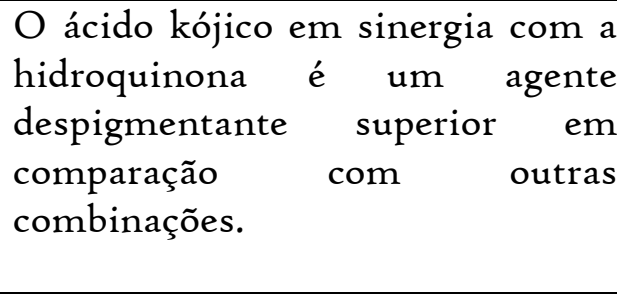 \\
\hline $\begin{array}{l}\text { HANDEL. A C. / } \\
2013\end{array}$ & $\begin{array}{l}\text { Avaliar os fatores de risco } \\
\text { para o desenvolvimento de } \\
\text { melasma facial em } \\
\text { mulheres. }\end{array}$ & $\begin{array}{l}\text { O melasma é associado à } \\
\text { capacidade de pigmentação da pele, } \\
\text { ancestralidade indígena, exposição } \\
\text { solar, estímulos hormonais e traços } \\
\text { de ansiedade. }\end{array}$ \\
\hline $\begin{array}{l}\text { IKINO, J. K. et al./ } \\
2015\end{array}$ & $\begin{array}{l}\text { Avaliar o impacto na } \\
\text { qualidade de vida de } \\
\text { mulheres por meio de } \\
\text { questionário (MelasQol), e } \\
\text { investigar os aspectos } \\
\text { clínicos e fatores de risco } \\
\text { para melasma. }\end{array}$ & $\begin{array}{l}\text { Início do melasma esteve associado } \\
\text { à gravidez. A análise revelou } \\
\text { impacto emocional significativo } \\
\text { sobre pacientes, como sentir-se } \\
\text { incomodado, frustrado, } \\
\text { constrangido e deprimido pela } \\
\text { aparência da pele. }\end{array}$ \\
\hline $\begin{array}{l}\text { MARTINS, V.C. } \\
\text { dos S.; OLIVEIRA, } \\
\text { S. P. de/ } 2015\end{array}$ & $\begin{array}{l}\text { Verificar a eficácia do } \\
\text { tratamento do melasma com } \\
\text { o ácido kójico associado ao } \\
\text { ácido glicólico. }\end{array}$ & $\begin{array}{l}\text { Existe eficácia da associação do } \\
\text { ácido kójico e ácido glicólico no } \\
\text { tratamento, porém são necessários } \\
\text { mais estudos. }\end{array}$ \\
\hline $\begin{array}{l}\text { MEDEIROS, J. K. } \\
\text { G. et al./ } 2016\end{array}$ & $\begin{array}{l}\text { Apresentar as eficácias do } \\
\text { tratamento combinado a } \\
\text { uma paciente com melasma. }\end{array}$ & $\begin{array}{l}\text { O ácido glicólico é o mais utilizado, } \\
\text { pois tem boa penetração na pele. A } \\
\text { tretinoína mostra-se tão eficiente } \\
\text { quanto o ácido glicólico e com } \\
\text { menos efeitos colaterais. }\end{array}$ \\
\hline $\begin{array}{lr}\text { MOTA, } & \text { J.P., } \\
\text { PAULO } & \text { ROXO } \\
\text { BARJA, P.R,/ } 2012\end{array}$ & $\begin{array}{l}\text { Classificar os tipos de pele } \\
\text { através de medidas } \\
\text { fotoacústicas in vivo, } \\
\text { relacionando o nível de sinal } \\
\text { fotoacústico à classificação } \\
\text { proposta por Fitzpatrick. }\end{array}$ & $\begin{array}{l}\text { Sob exposição à radiação solar, a } \\
\text { pele (sinal fotoacústico) sofre } \\
\text { homogeneização, dificultando a } \\
\text { observação de diferenças entre os } \\
\text { fototipos. }\end{array}$ \\
\hline
\end{tabular}




\begin{tabular}{|c|c|c|}
\hline $\begin{array}{l}\text { MOURA, M, C. de; } \\
\text { et al. / } 2017\end{array}$ & $\begin{array}{l}\text { Analisar a eficácia da ação } \\
\text { combinada } \\
\text { microagulhamento } \\
\text { associado a ativos } \\
\text { clareadores no tratamento } \\
\text { de manchas hipercrômicas. }\end{array}$ & $\begin{array}{l}\text { O protocolo aplicado com } \\
\text { microagulhamento associado a } \\
\text { ácidos e ativos clareadores, com } \\
\text { Vitamina } C \text { e uso diário de FPS } 70 \text {, } \\
\text { mostrou-se eficaz no tratamento } \\
\text { das manchas hipercrômicas. }\end{array}$ \\
\hline $\begin{array}{l}\text { NOLASCO, I.M.L.; } \\
\text { RESENDE, J. R./ } \\
2020\end{array}$ & $\begin{array}{l}\text { Discutir a ação do peeling } \\
\text { mandélico como ativo } \\
\text { despigmentante. }\end{array}$ & $\begin{array}{l}\text { O ácido mandélico ainda é pouco } \\
\text { utilizado em comparação a outros } \\
\text { ácidos mais agressivos como o } \\
\text { retinóico e o glicólico, o que } \\
\text { ressalta a importância dos estudos } \\
\text { acerca do tema abordado. }\end{array}$ \\
\hline $\begin{array}{l}\text { OLIVEIRA. G. C. } \\
\text { de,;PEREIRA, G. G.; } \\
\text { CERRI, M.F. / 202I }\end{array}$ & $\begin{array}{l}\text { Realizar um levantamento } \\
\text { bibliográfico sobre a } \\
\text { aplicabilidade dos peelings } \\
\text { químicos na estética. }\end{array}$ & $\begin{array}{l}\text { Os ácidos que podem ser aplicados } \\
\text { nos procedimentos de peelings } \\
\text { químicos: glicólico, mandélico, } \\
\text { retinóico, salicílico, ascórbico } \\
\text { (vitamina C), lático e fenol. }\end{array}$ \\
\hline
\end{tabular}

Fonte: elaborado pelos

Tabela IC: Síntese dos estudos selecionados para revisão.

\begin{tabular}{|c|c|c|}
\hline Autor/Ano & Objetivo(s) & Resultados/Conclusão \\
\hline $\begin{array}{l}\text { PEDROSA, } \\
\text { do N./ } 2013\end{array}$ & $\begin{array}{l}\text { Estudar o potencial de } \\
\text { derivados do ácido kójico, } \\
\text { visando obter compostos com } \\
\text { atividades despigmentantes, } \\
\text { antioxidantes } \\
\text { rejuvenescedoras. }\end{array}$ & $\begin{array}{l}\text { A ação sobre a melanogênese do } \\
\text { kojato de cobre e oleikojato de cobre } \\
\text { ocorrem pela inibição da tirosinase } \\
\text { associada a um mecanismo } \\
\text { antioxidante, tendo potencial como } \\
\text { agentes despigmentantes. }\end{array}$ \\
\hline $\begin{array}{l}\text { PONTES, C. } \\
\text { G.; MEJIA, D. } \\
\text { P. M. / 20I4 }\end{array}$ & $\begin{array}{l}\text { Organizar dados e evidências } \\
\text { que contribuam com a eficácia } \\
\text { do ácido kójico no tratamento } \\
\text { de melasma. }\end{array}$ & $\begin{array}{l}\text { O ácido kójico é um dos mais } \\
\text { eficientes do mercado, tendo } \\
\text { excelentes resultados, pois não causa } \\
\text { irritação e nem fotossensibilização ao } \\
\text { usuário. }\end{array}$ \\
\hline $\begin{array}{l}\text { PURIM, K. S. } \\
\text { M.; AVELAR, } \\
\text { M. F. DE S./ } \\
2012\end{array}$ & $\begin{array}{l}\text { Avaliar hábitos de exposição } \\
\text { solar e fotoproteção entre } \\
\text { gestantes, presença de } \\
\text { melasma e seu impacto na } \\
\text { qualidade de vida. }\end{array}$ & $\begin{array}{l}\text { O melasma gera desconfortos } \\
\text { socioemocionais, com queixas da } \\
\text { aparência da pele, frustração e } \\
\text { constrangimento. }\end{array}$ \\
\hline $\begin{array}{l}\text { RODRIGUES, } \\
\text { A. M. da S./ } \\
2014\end{array}$ & $\begin{array}{l}\text { Proporcionar uma abordagem } \\
\text { farmacêutica adequada a } \\
\text { pessoas atingidas por } \\
\text { hiperpigmentação, bem como } \\
\text { o desenvolvimento de novas } \\
\text { terapias. }\end{array}$ & $\begin{array}{l}\text { O uso isolado de ácido retinóico } \\
\text { reduziu o melasma, porém, em altas } \\
\text { concentrações, implica em resultados } \\
\text { indesejáveis. O ácido glicólico, ácido } \\
\text { kójico e a vitamina C, demonstraram } \\
\text { bons resultados, como ativos ou } \\
\text { coadjuvantes de outros } \\
\text { despigmentantes. }\end{array}$ \\
\hline
\end{tabular}




\begin{tabular}{|c|c|c|}
\hline $\begin{array}{l}\text { SANTOS, } \\
\text { S.L.F. dos et al./ } \\
2017\end{array}$ & $\begin{array}{l}\text { Abordar as contraindicações } \\
\text { do uso de peeling químico. }\end{array}$ & $\begin{array}{l}\text { A aplicação realizada de forma } \\
\text { inadequada sobre a pele inflamada, } \\
\text { com queimaduras de sol ou a } \\
\text { utilização com } \\
\text { outros tratamentos tópicos, podem } \\
\text { ocasionar efeitos dermatológicos } \\
\text { graves. }\end{array}$ \\
\hline $\begin{array}{l}\text { SCHAEFER, L. } \\
\text { V./2018 }\end{array}$ & $\begin{array}{l}\text { Identificação diferencial de } \\
\text { proteínas expressas na pele } \\
\text { com melasma facial e na pele } \\
\text { sã adjacente. }\end{array}$ & $\begin{array}{l}\mathrm{Na} \text { pele com melasma foram } \\
\text { identificadas proteínas que } \\
\text { participam de funções ligadas à } \\
\text { glicólise, gliconeogênese, transporte } \\
\text { celular, hemostasia, coagulação, } \\
\text { cicatrização e estímulos externos. }\end{array}$ \\
\hline $\begin{array}{l}\text { SILVA, L. S.; } \\
\text { MONTEIRO, } \\
\text { M. S. S. B. / } \\
2016\end{array}$ & $\begin{array}{l}\text { Avaliar a segurança das NPs } \\
\text { de } \mathrm{ZnO} \text { e } \mathrm{TiO}_{2} \text {, utilizadas } \\
\text { como ativos fotoprotetores. }\end{array}$ & $\begin{array}{l}\text { Os } \mathrm{NPs} \text { de } \mathrm{TiO}_{2} \text { e } \mathrm{ZnO} \text { podem ser } \\
\text { seguras, são necessários estudos sobre } \\
\text { a segurança dessas NPs. }\end{array}$ \\
\hline $\begin{array}{l}\text { SPADAFORA, } \\
\text { M.C.F. de A. } \\
\text { /2019 }\end{array}$ & $\begin{array}{l}\text { Discutir sobre os benefícios da } \\
\text { utilização dos } \\
\text { despigmentantes para o } \\
\text { tratamento de melasmas e } \\
\text { rejuvenescimento facial. }\end{array}$ & $\begin{array}{l}\text { Grande uso em forma de gel, creme, } \\
\text { sérum, loções e na aplicação de } \\
\text { peeling químico para os tratamentos } \\
\text { da pele, com resultados eficientes. }\end{array}$ \\
\hline $\begin{array}{l}\text { SOUZA, L. C. } \\
\text { de, AMURIM, } \\
\text { N. P., } \\
\text { GRIGNOLI, L. } \\
\text { C. M. E./ 2018 }\end{array}$ & $\begin{array}{l}\text { Descrever a origem do } \\
\text { melasma e avaliar a eficácia do } \\
\text { tratamento da hipercromia } \\
\text { utilizando o ácido kójico } \\
\text { associado ao ácido glicólico. }\end{array}$ & $\begin{array}{l}\text { O uso associado do ácido kójico e } \\
\text { glicólico é eficiente no tratamento do } \\
\text { melasma, apresentando favoráveis } \\
\text { resultados em comparação com a } \\
\text { hidroquinona, por não apresentar } \\
\text { reações adversas ao usuário. }\end{array}$ \\
\hline $\begin{array}{lr}\text { TARAZ, } & \text { M; } \\
\text { NIKNAM, } & \text { S; } \\
\text { EHSANI, } & \\
\text { A.H. / 2017 } & \\
\end{array}$ & $\begin{array}{l}\text { Avaliar o uso do laser e do } \\
\text { ácido tranexâmico no } \\
\text { tratamento do melasma. }\end{array}$ & $\begin{array}{l}\text { O ácido tranexâmico usado com o } \\
\text { laser demostrou que sua eficácia não é } \\
\text { estabelecida de forma adequada. }\end{array}$ \\
\hline $\begin{array}{l}\text { URASAKI, M. } \\
\text { B. M./ } 2018\end{array}$ & $\begin{array}{l}\text { Analisar os conhecimentos, } \\
\text { atitudes e práticas da equipe de } \\
\text { saúde sobre melasma na } \\
\text { gravidez. }\end{array}$ & $\begin{array}{l}\text { As pacientes desconhecem as causas, } \\
\text { prevenção e tratamento do melasma, } \\
\text { além de considerar que as manchas na } \\
\text { pele não interferem na qualidade de } \\
\text { vida, e a condição é considerada um } \\
\text { problema simples. }\end{array}$ \\
\hline
\end{tabular}

\section{DISCUSSÃO}

Os artigos selecionados para este estudo abordaram pontos importantes sobre o tratamento do melasma, principalmente sobre o uso de diferentes tipos de ácidos utilizados nos procedimentos químicos e os problemas socioemocionais relatados nas pesquisas. 
O melasma é conceituado como uma doença crônica da pele, caracterizada por uma hiperpigmentação da área dérmica exposta ao sol (COLFERAI et al., 2018). Além disso, pesquisas demonstram resultados que afirmam a influência decisiva da exposição à radiação solar na pele, uma vez que para se proteger da irradiação solar, a pele utiliza um mecanismo específico e complexo denominado pigmentação (MOTA; BARJA, 2002).

Embora haja uma discussão sobre a exposição da pele ao sol, o melasma é constantemente associado à gravidez, alterações hormonais, anticoncepcionais orais, hepatopatias e uso de drogas cosméticas (TARAZ et al., 2017). Estudos relatam que 22,9\% das mulheres entrevistadas em sua pesquisa possuíam melasma, sendo que $80 \%$ desenvolveram manchas durante a gestação (PURIM; AVELAR, 20I2).

Saliente-se ainda que, o uso de anticoncepcionais orais no período da gravidez e a reposição de hormônios são apontados como indicadores hormonais na evolução do melasma (SCHAEFER, 2018). Contudo, resultados de pesquisas mostraram que os pacientes com melasma tiveram um aumento do quadro de ansiedade, como também um acréscimo no nível de estresse e transtornos depressivos, sendo que esses fatores estão ligados ao aumento da produção de cortisol e melanocortina, que estão envolvidos na atividade melanogênica (HANDEL, 2013).

As características genéticas identificadas em conjunto com a influência de fototipos, ancestralidade e história familiar sobre o paciente com melasma devem ser estudadas para compreensão do tipo de tratamento e controle adequados (HANDEL, 2013). A classificação clínica usual proposta por Fitzpatrick (1975), baseia-se pela observação visual da pele, que a classifica de acordo ao fototipo e etnia, distribuídos do tipo I (pele mais branca) ao tipo VI (pele negra), identificando o eritema e a pigmentação adquirida após a exposição à radiação ultravioleta (Tabela 2).

Figura r: Classificação de Fitzpatrick para Fototipos de Pele

\begin{tabular}{|c|c|c|c|c|}
\hline Fototipo & Aparência & $\begin{array}{c}\text { Reação à exposição } \\
\text { solar }\end{array}$ & $\begin{array}{c}\text { Pigmentação } \\
\text { imediata } \\
(6-8 \text { horas })\end{array}$ & $\begin{array}{c}\text { Pigmentação } \\
\text { retardada (10-I4 } \\
\text { dias) }\end{array}$ \\
\hline I & $\begin{array}{c}\text { Pele muito } \\
\text { branca, cabelos } \\
\text { loiros ou ruivos, } \\
\text { olhos claros e } \\
\text { frequentemente } \\
\text { sardas }\end{array}$ & $\begin{array}{c}\text { Queima com } \\
\text { facilidade, porém } \\
\text { nunca bronzeia }\end{array}$ & Nenhuma & Nenhuma \\
\hline
\end{tabular}


-

\begin{tabular}{|c|c|c|c|c|} 
II & $\begin{array}{c}\text { Pele clara, olhos } \\
\text { claros, cabelo } \\
\text { claro }\end{array}$ & $\begin{array}{c}\text { Queima facilmente, } \\
\text { bronzeia muito pouco }\end{array}$ & Fraca & Poúnima à fraca \\
\hline III & $\begin{array}{c}\text { Pele clara, olhos } \\
\text { e cabelo de cor } \\
\text { variável }\end{array}$ & $\begin{array}{c}\text { Queima um pouco, } \\
\text { bronzeia } \\
\text { gradualmente }\end{array}$ & Baixa \\
\hline IV & $\begin{array}{c}\text { Pele } \\
\text { moderadamente } \\
\text { pigmentada a } \\
\text { muito } \\
\text { pigmentada }\end{array}$ & $\begin{array}{c}\text { Raramente queima e } \\
\text { bronzeia com } \\
\text { facilidade }\end{array}$ & Moderada & Moderada \\
\hline V & $\begin{array}{c}\text { Escura ou do } \\
\text { sudoeste asiático }\end{array}$ & $\begin{array}{c}\text { Não queima e } \\
\text { bronzeia }\end{array}$ & Intensa & Intensa \\
\hline VI & $\begin{array}{c}\text { Muito escura } \\
\text { Bronzeia facilmente }\end{array}$ & Muito intensa & Intensa \\
\hline
\end{tabular}

Fonte: Adaptado da Sociedade Brasileira de Dermatologia (2021) e Food Drug Administration (2021).

As formas de tratamento de manchas de pele dependem de uma análise minuciosa da lesão a ser tratada, a origem do problema e o fototipo de pele, para se alcançar um tratamento mais efetivo (ARAÚJO; MEIJA, 2014).

\section{TRATAMENTO PARA O MELASMA}

Vários estudos abordam que nenhum tratamento existente para o melasma pode ser considerado mais eficaz que o outro (CUNHA; SILVA; e OLIVEIRA, 2020). Inclusive, autores afirmam que o uso de diferentes terapias tópicas com seus distintos mecanismos de ação, fornece resultados positivos no tratamento das hipercromias (NOLASCO; RESENDE, 2020).

Para o tratamento do melasma é evidenciado o uso de terapias combinadas, como: peelings químicos e laser, ou microagulhamento e peeling, ou microagulhamento com ativos despigmentantes (BORGES, 2021; MOURA, 2017) C Contudo, para cada tipo de pele existe um protocolo de tratamento, onde leva-se em deferência características de sensibilidades da derme (NOLASCO; RESENDE, 2020). 
O uso de ativos combinados proporciona a suavização da textura da pele e a remoção das camadas exteriores que estão danificadas, com isso, há a destruição de forma regulada da epiderme e da derme, que é seguido por um processo regenerativo, sendo esta alternativa indicada no tratamento de patologias estéticas, como o melasma (MOURA, 2017).

Os ácidos conseguem proporcionar a esfoliação da pele ao tornarem-na ácida, pois estes possuem um $\mathrm{pH}$ inferior, sendo assim é produzida uma lesão controlada e com isso, obtémse a diminuição de discromias pigmentares (BORGES, 202I).

Cada substância atua por meio de um mecanismo diferente, o que causa resultados e efeitos colaterais específicos. Diante disso, destaca-se a seguir os três principais ácidos dermocosméticos utilizados no tratamento do melasma:

\section{ÁCIDO GLICÓLICO}

Classificado como um alfa-hidroxiácido, o ácido glicólico é considerado o principal agente esfoliante utilizado no tratamento terapêutico do melasma (MOURA, 2017). Dessa maneira, o ácido glicólico, por afinar o estrato córneo e reduzir a coesão entre os corneócitos, é indicado para o tratamento de hipercromias (aumento da melanina), especialmente quando associado à despigmentantes (MARTINS; OLIVEIRA, 2015).

O tratamento com ácido glicólico em pacientes ambulatoriais apresenta resultados positivos, pois o ácido glicólico possui menor peso molecular, facilitando a sua penetração, baixo poder de fotossensibilização e capacidade de provocar respostas imunológicas, quando comparado a outros alfa-hidroxiácidos (SPADAFORA, 2019).

As concentrações do ácido glicólico variam de acordo com a resistência da pele e o objetivo do tratamento, pois ao ser colocado em um $\mathrm{pH}$ equivalente a 3,8 terá ação esfoliante e despigmentante, porém ao ser colocado em $\mathrm{pH}$ equivalente a 6,o transforma-se em glicolato, sendo considerado um ótimo agente hidratante, sendo assim, quanto mais o $\mathrm{pH}$ se eleva, reduz-se a habilidade de renovação da pele (PEREIRA, 2013).

Contudo, o ácido glicólico é muito utilizado em tratamentos de melasma, em razão ao uso da porcentagem correta de concentração em conformidade ao tipo de pele e da espessura da camada córnea (OLIVEIRA; PEREIRA; e CERRI, 202I). Ainda, relatam sobre os efeitos das concentrações, que para apresentarem o efeito esfoliante-descamante, devem apresentar altas concentrações, variando entre $6-20 \%$. No entanto, para efeito de plasticidade e 
abrasão, as concentrações devem ser menores que 6\% (OLIVEIRA; PEREIRA; e CERRI, 2021).

O ácido glicólico é combinado com outros agentes a uma concentração de 5 a Io\% por sua propriedade de clarear a pele, sendo que o mecanismo de seu efeito pode ser devido à remodelação epidérmica e descamação acelerada, o que resultaria em rápida dispersão do pigmento nas lesões pigmentares e também reduz diretamente a formação de melanina nos melanócitos pela inibição da tirosinase (OLIVEIRA; PEREIRA; e CERRI, 202I).

Em síntese, o ácido glicólico é muito utilizado em produtos dermatológicos, como esfoliantes químicos e antirrugas, no entanto, é importante ter cuidado com a utilização desses tipos de cosméticos, pois podem causar desde inchaço até a descoloração da pele (CENTURIÃO, 2020).

\section{ÁCIDO KÓJICO}

O ácido kójico, 5-hidroxi-2-hidroximetil-4-piranona, é utilizado no Japão para tratar a hiperpigmentação desde 1989, devido ao seu efeito inibitório da tirosinase, o que resulta na diminuição da síntese de melanina e induz a diminuição da eumelanina em células hiperpigmentadas (PONTES; MEJIA, 2014).

A aplicação do ácido kójico para tratamentos de hiperpigmentação se dá por meio de emulsões manipuladas em farmácias magistrais, evidenciando que o fármaco entra em contato com as células, permeando pelo organismo até atingir o local a ser tratado, inibindo a tirosinase (PEDROSA, 2013). Contudo, o ácido kójico, mostra resultados positivos, tanto como ativo principal ou coadjuvante de outros despigmentantes (RODRIGUES, 2014).

A tênue ação do ácido kójico na pele é a vantagem desse ativo nos tratamentos do melasma, pois o mesmo não causa irritação e nem fotossensibilização (SOUZA; AMURIM; e GRIGNOLI, 2018). Para o desenvolvimento adequado do tratamento, é necessário que a pele esteja totalmente higienizada, assim haverá uma excelente absorção do despigmentante, uma vez que o ácido kójico não é um ácido citotóxico e que também pode ser facilmente associado com outros agentes clareadores (PONTES; MEJIA, 2014).

Portanto, o ácido kójico é visto como um dos despigmentantes naturais mais eficazes do mercado, tendo excelentes resultados descritos em trabalhos e com importante colocação 
de produtos com essa substância no mercado dos dermocosméticos, consistindo nos mais usados no clareamento de vários tipos de hipercromias cutâneas (MOURA, 2017).

Em um estudo comparativo simples randomizado (números aleatórios gerados por computador), realizado com um grupo de 80 adultos saudáveis de ambos os sexos com diagnóstico clínico de melasma, constataram que nos casos onde foram aplicados o ácido kójico à $2 \%$ junto com a hidroquinona à $2 \%$, os pacientes tiveram uma resposta melhor do que aqueles que aplicaram apenas hidroquinona, comprovando a sinergia destas duas substâncias e seu efeito clareador (DEO et al., 2013).

Contudo, a hidroquinona com concentrações maiores que 2\%, apesar de promover bons resultados, causa efeitos citotóxicos, resultando em irritações cutâneas (SOUZA; AMURIM; e GRIGNOLI, 2018). Sendo assim, o ácido kójico e o ácido glicólico associados no tratamento do melasma demonstraram melhores resultados e menos efeitos colaterais nos pacientes (RODRIGUES, 2014).

\section{ÁCIDO MANDÉLICO}

Um dos métodos mais eficazes de tratamento do melasma são os peelings químicos contendo ácido mandélico, que age pela despigmentação e reduz o trabalho dos melanócitos (CUNHA; SILVA; e OLIVEIRA, 2020), pois a hiperpigmentação é uma das principais queixas de pacientes em procedimentos cosméticos.

As substâncias ativas, como os alfahidroxiácidos (AHAs), especificamente o ácido mandélico, tem como indicações principais casos de hiperpigmentação, acne inflamatória não cística e rejuvenescimento em peles fotoenvelhecidas. Outrossim, o ácido mandélico atua promovendo a despigmentação através da inibição da síntese de melanina (SOUZA; AMURIM; e GRIGNOLI, 2018).

O ácido mandélico é um composto químico de alto peso molecular de absorção lenta pela derme, com um efeito uniforme, que pode ser obtido do extrato de amêndoas amargas. É bastante utilizado para combater hiperpigmentações, além de melhorar a textura da pele, agindo na inibição da síntese de melanina, bem como na melanina já depositada (PEREIRA; MEJIA, 2014).

Usado como um agente clareador e eficaz, o ácido mandélico consegue romper o cimento intercelular, criando descamação e conferindo uma aparência homogênea à pele, pois é uma substância atóxica e menos irritante, com grande vantagem de não reagir ao sol, 
sendo uma alternativa para pacientes que precisam cuidar da pele continuamente (NOLASCO; RESENDE, 2020).

Embora seja considerado um ácido seguro para os fototipos indicados na escala de Fitzpatrick (FITZPATRICK, 1975), este deve ser usado de forma controlada e com orientação de um profissional habilitado, pois pelo fato de ser um produto químico, podem ocorrer efeitos colaterais, como: eritema, inchaço, ardor, bolhas, sangramento, erupção cutânea e coceira (NOLASCO; RESENDE, 2020).

\section{PREVENÇÃO E QUALIDADE DE VIDA}

A ação dos raios solares é um dos principais fatores de risco para os problemas de pele. Dessa maneira, é importante adotar métodos para a prevenção do melasma, como mudança de hábitos e a utilização de protetores solares (IKINO, 20I5). Os filtros solares físicos ou químicos possuem uso tópico e diferenciam-se em seu mecanismo de ação pela composição das suas moléculas ativas, sendo classificados em inorgânicos ou (físicos) e orgânicos ou (químicos) (PURIM; AVELAR, 2012).

As formulações antissolares contendo filtros solares físicos apresentam uma característica sensorial gordurosa, devido à alta carga lipídica adicionada à formulação, para que esta seja resistente à água. Portanto, há a formação de um filme esbranquiçado e opaco sobre a pele, que acaba refletindo a radiação UV e a luz visível. Os fotoprotetores inorgânicos são compostos por minerais ativos como o óxido de zinco e o dióxido de titânio com propriedades fotocatalíticas, que formam uma barreira física na pele capaz de refletir e dispersar os raios UV e visível (SILVA; MONTEIRO, 2016).

O método de fotoproteção química é formado por uma associação de substâncias químicas que são capazes de barrar as radiações UVA e UVB. Portanto, a proteção solar é vital para o sucesso de qualquer tratamento prescrito, variando entre os tipos de protetores, que vão desde formulações físicas às formulações químicas, com mecanismos moleculares de reparo celular (MEDEIROS, 2016).

Recomenda-se que o protetor solar seja aplicado meia hora antes da exposição solar e, se caso necessário, seja feito o uso de roupas e acessórios com mangas longas, chapéu, óculos de sol e guarda sol. Além disso, deve-se evitar a exposição no horário de pico, entre ioh às I6h, e procurar sombra e lugares frescos (PURIM; AVELAR, 2012). 
O melasma afeta de forma negativa a qualidade de vida do indivíduo, já que acomete áreas visíveis do corpo como o rosto, tornando dificultosa a aceitação da própria aparência, o que gera uma baixa autoestima. As manchas evidentes podem provocar certo constrangimento durante o convívio social, além de se tornarem um obstáculo em determinados casos no âmbito profissional (HANDEL, 2013).

Por provocar mudanças na aparência, o indivíduo sofrerá com alterações e limitações em seu cotidiano que não contribuem para o seu bem-estar (URASAKI, 2018; SANTOS et al., 2017). Desse modo, com o intuito de usufruir de uma melhoria na sua qualidade de vida, o paciente deve buscar um tratamento eficaz que lhe proporcione benefícios como o clareamento das lesões, a prevenção de complicações relacionadas à exposição à radiação ultravioleta, e que potencialize o nível de satisfação com a imagem corporal, além de conferir a este o bem-estar emocional (MARTINS; OLIVEIRA, 2015).

\section{CONSIDERAÇÕES FINAIS}

O fotoenvelhecimento da pele é um processo dinâmico e progressivo motivado por vários fatores intrínsecos e extrínsecos. Neste estudo, observou-se que o gerenciamento do melasma pode ser desafiador e requer tratamento a longo prazo com agentes tópicos.

O conhecimento amplo e aprofundado sobre a estrutura e função da pele permitiu o desenvolvimento de produtos para seu cuidado, com apreciáveis efeitos benéficos. Tais avanços conduziram ao desenvolvimento de produtos cada vez melhores para prevenção e tratamento do melasma, através de terapia combinada, que é a modalidade de tratamento preferida para o sinergismo e a redução de efeitos indesejáveis.

Logo, o estudo permitiu compreender que o uso de fotoprotetores para a prevenção, juntamente com o tratamento facial, tendo como base o ácido glicólico, ácido kójico e ácido mandélico, trouxeram resultados importantes que contribuíram para o rejuvenescimento e clareamento da pele, uma vez que o melasma não possui cura. Todavia, o controle desta patologia é essencial para a satisfação com a autoimagem e para que se desfrute de uma boa qualidade de vida.

\section{REFERÊNCIAS}

ARAÚJO, Islane Lunier; MEJIA, Dayana Priscila Maia. Peeling químico no tratamento das hipercromias. Faculdade Cambury Bio Cursos, 2014. 
BENTO, Antônio. Como fazer uma revisão da literatura: considerações teóricas e práticas. Revista JA, 7(65), 42-44, 2012.

BORGES, Fernando Gomes; PEREIRA, Tatiana Aparecida. Avaliação do comportamento do consumidor frente ao consumo de dermocosméticos em Araxá-MG, TCC (graduação em Farmácia) - Universidade de Uberaba, Minas Gerais, 32 f., 2018.

BORGES, Maysa Coelho. Melasma: tratamento e suas implicações estéticas. Health of Humans, v. 3, n. I, p. 8-19, 2021.

BRIANEZI, Gabrielli. Avaliação da atividade da unidade epidermo-melânica e do dano dérmico no melasma. 20I6. (Tese de doutorado) Universidade Estadual Paulista "Júlio de Mesquita Filho”. Escola: Faculdade de Medicina de Botucatu - Curso de Graduação de Enfermagem. 2016.

CENTURIÃO, Patrícia de Oliveira et al. Avaliação da qualidade de produtos cosméticos contendo ácido glicólico. Revista Colombiana de Ciências Químico-Farmacêuticas, v. 5o, n. I, 2020.

CESÁRIO, Gleiciane Rodrigues. Principais ativos utilizados no tratamento do melasma. 2015. 55 f. Trabalho de Conclusão de Curso (Graduação) - Curso de Farmácia, Centro Universitário Luterano de Palmas (CEULP/ULBRA), Palmas-TO, 2015. 
CESTARI, Tania Ferreira; DANTAS, Lia Pinheiro; BOZA, Juliana Catucci. Acquired hyperpigmentations. Revista Anais Brasileiros de Dermatologia. v. 89, p. II-25, 2014.

COLfERAI, Mariana Morais Tavares; MIQUELIN, Gabriela Momente; STEINER, Denise. Evaluation of oral tranexamic acid in the treatment of melasma. Journal of Cosmetic Dermatology v.78, n.2, p.363-369, 2018.

CUNHA, Isadora Gonçalves; SILVA, Claudia Peres; OLIVEIRA, Geraldo. Principais tratamentos do melasma. Humanidades e tecnologia (FINOM), v. I, n. 23, p. 302-315, 2020.

DEO, Kirti et al. Kojic acid vis-a-vis its combinations with hydroquinone and betamethasone valerate in melasma: a randomized, single blind, comparative study of efficacy and safety. Indian Journal of Dermatology, 2013;58(4):28I-285.

FITZPATRICK, Thomas Bernard. (1975). "Soleil et peau" [Sun and skin]. Journal de Médecine Esthétique (em francês) (2): 33-34, US Food \& Drug Administration. Your skin. https://www.fda.gov/radiation-emitting-products/tanning/your-skin (acessado em or de outubro de 2021).

HANDEL, Ana Carolina. Fatores de risco para melasma facial em mulheres: um estudo caso-controle. Dissertação (Mestrado em Patologia) - Faculdade de Medicina de Botucatu, Universidade Estadual Paulista "Júlio de Mesquita Filho", Botucatu, p. Ioo, 2013.

IKINO, Juliana Kida et al. Melasma and assessment of the quality of life in Brazilian women. Anais brasileiros de dermatologia, v. 90, p. 196-200, 2015. 
MACEDO, Juliana Rodrigues Bueno. 2019. Fisiopatologia do Melasma. NEPUGA Núcleo de Estudos e Treinamento Ana Carolina Puga. Pós-graduação em Biomedicina Estética. São Paulo, 2019.

MARTINS, Vanessa Cristiane dos Santos; OLIVEIRA, Silvia Patricia. Estudo dos benefícios do tratamento de melasma por intermédio do ácido kójico associado ao ácido glicólico. 2015. 13 f. Monografia (Especialização) - Curso de Mba em Estética Clínica Avançada e Cosmetologia, Universidade Tuiuti do Paraná, Tuiut, 2015.

MEDEIROS, Janielle Kelly Guimarães et al. Combinação terapêutica no tratamento do melasma. Cuidarte Enfermagem, v.ıo, n.2, 2016.

MOTA, Jociely; BARJA, Paulo Roxo. Classificação dos fototipos de pele: análise fotoacústica vesus análise clínica. X Encontro Latino Americano de Iniciação Científica e VI Encontro Latino Americano de Pós-Graduação - Universidade do Vale do Paraíba, p. $2561-2564,2002$.

MOURA, Maria Cristiana et al. $\mathrm{O}$ uso de ácidos e ativos clareadores associados ao microagulhamento no tratamento de manchas hipercrômicas: estudo de caso. Revista Científica da FHO, UNIARARAS, v. 5, n. 2, 2017.

NOLASCO, Izis Moara Morais Leão; RESENDE, Juliana Resende. Uso do ácido mandélico no tratamento de hipercromias pós-inflamatória: uma revisão de literatura. Scire Salutis, v.1o, n.2, p.35-42, 2020. 
OLIVEIRA, Gheisa Carla; PEREIRA, Giorgia Gomes; CERRI, Murilo Fanchiotti. Aplicabilidade dos peelings químicos: revisão de literatura. Revista Acadêmica Novo Milênio. V 3. N 4. 2021.

PEDROSA, Tatiana do Nascimento. Avaliação do potencial de derivados do ácido kójico sobre a melanogênese e o envelhecimento cutâneo. 2013. II4 f. Dissertação (Mestrado em Ciências Farmacêuticas) - Universidade Federal do Amazonas, Manaus, 2013.

PEREIRA, Ana Mirte Viana; MEJIA, Dayana Priscila Maia. Peelings químicos no rejuvenescimento facial. Pós-graduação em Fisioterapia em Dermato-Funcional, Faculdade Cambury. 20I4.

PEREIRA, Maria de Fátima Lima. Cosmetologia. i ed. São Caetano, do Sul, SP: Editora Difusão, 2013.

PONTES, Caroline Gomes; MEJIA, Dayana Priscila Maia. Ácido Kójico no Tratamento do Melasma. 2014. I2 f. Dissertação (Mestrado) - Curso de Dermatofuncional, Faculdade Cambury, Goiânia, 2014.

PURIM, Kátia Sheylla Malta; AVELAR, Maria Fernanda de Santana. Fotoproteção, melasma e qualidade de vida em gestantes. Revista Brasileira de Ginecologia e Obstetrícia, v. 34, p. 228-234, 2012 .

RODRIGUES, Ana Miguel da Silva. Hiperpigmentação - moléculas de tratamento inovadoras. 3I f. Dissertação (Mestrado Integrado em Ciências Farmacêuticas) Universidade de Coimbra, Coimbra, Portugal, 2014. 
SANTOS, Sandna Larissa Freitas et al. Peelings químicos: contraindicações e complicações dermatológicas. Mostra Científica da Farmácia, Araxá, v. 2, n. 2, 2017.

SCHAEFER, Luiza Vasconcelos. Estudo proteômico do melasma facial em mulheres. 2018. (Dissertação) Mestrado. Universidade Estadual Paulista "Júlio de Mesquita Filho" Faculdade de Medicina”. 2018.

SILVA, Lívia Sousa; MONTEIRO, Mariana Sato. Avaliação da Segurança de Nanopartículas de Dióxido de Titânio e Óxido de Zinco em Formulações Antissolares. Revista Virtual de Química, v. 8, n. 6, p. 1963-1967, 2016.

Sociedade Brasileira de Dermatologia. Classificação dos fototipos de pele. https://www.sbd.org.br/dermatologia/pele/cuidados/classificacao-dos-fototipos-de-pele/ (acessado em or de outubro de 202I).

SOUZA, Leticia Carvalho; AMURIM, Nathália Pereira; GRIGNOLI, Laura Cristina Marretto Esquissato. O Uso Associado do Ácido Kójico e Ácido Glicólico como Alternativa à Hidroquinona no Tratamento de Melasma. Revista Científica Multidisciplinar Núcleo do Conhecimento, v.2, 2018.

SPADAFORA, Maria Claudia Fonseca de Almeida et al. Os benefícios dos despigmentantes para o tratamento do melasma e rejuvenescimento facial. Revista Saúde em Foco, n. II, 2019.

TARAZ, Mohammad; NIKNAM, Somayeh; EHSANI, Amir Houshang. Tranexamic acid in treatment of melasma: A comprehensive review of clinical studies. Dermatologic therapy, v. 30, n. 3, p. e12465, 2017. 
URASAKI, Maristela Belletti Mutt. Conhecimento, atitude e prática da equipe de saúde sobre melasma na gravidez. Avances en Enfermería, v. 36, n. I, p. 40-49, 2018. 